\title{
The VUCA Method for Leadership and Management in Times of Crisis EX. (Covid-19 \& Others Crisis: Practices, Lessons, and perspectives)
}

\author{
Mohammed Meri \\ University of Strasbourg- Laboratoire Sage, France
}

\begin{abstract}
The world is characterized by rapid change and data overload, technology is evolving at an accelerated pace, it is changing our lives today: creating and collecting knowledge, communicating them with others, traveling, music, shopping, consumption, and human relations; the consequences are enormous. Familiar concepts such as: (job, company, nationality, management, leadership, and culture) develop into a new reality which corresponds to the changes brought by the new world. Therefore, companies, state authorities and NGOs must operate in change at the same speed, and constantly adapt the products and services they offer, as well as the way they produce, promote, and sell them. These organizations are desperately trying to be proactive, to imagine what the world of tomorrow might look like and to continually develop new suitable products and services, as the world has become so unpredictable, testing and error has become one of the ways of economy. In times of crisis, they need to change more fundamentally because they need new business models, different organizational structures, different employee profiles, different leaders, and different ways of interacting with each other. This article discusses the Vuca method as a new approach to leadership and management "by six categories of the countries of the world " in times of crisis (Covid-19 and other crises), to achieve adaptation, performance and development of public administrations, businesses, NGOs, and civil society.
\end{abstract}

Keywords: The VUCA Method, Leadership \& Management, Times of Crisis 